\title{
A Comparative Study on Kinematic Performance of Corner Kicks for Junior Soccer Players According to Ball Rotation towards Effective Areas in Front of the Goal Dr/ Hassan Ibrahim Ali
}

\section{Abstract:}

In this study the research aim was to compare differences among kinematic properties such as displacement and linear velocities and angles and angular velocities during performing corner kicks for Junior Soccer players with techniques of ball rotation towards the goal in the close post area and to the contrary of the goal in the rear area at the moment of launching the ball. The study was carried out on (3) Junior players from Al-Sharkia Sporting Club under (18) years who were the best in playing corner kicks according to the test of corner kicks performance precision to specific areas in front of the goal with the right foot. (10) Trials were shot with video for each technique. To measure the precision of corner kicks according to ball rotation towards the goal technique the researcher depended on the test of the precision of passing the corner kick in front of the goal. Kinematic parameters were determined by using Simi Motion Program for motor analysis designed to track and to analyze the motion. Results indicated that there are significant differences in Displacements and linear (horizontal, cross and vertical) velocities of some body parts and the ball during performing corner kicks for the close post and beyond area according to ball rotation technique at the moment of launching the ball, and there are significant differences in Angles and angular velocities of body parts during performing corner kicks for the close post and beyond area according to ball rotation technique at the moment of launching the ball

Key words:

Kinematic, Corner Kicks, Junior Soccer Players, Ball Rotation, Simi Motion Program, Areas in Front of the Goal.

\section{Introduction}

Kicking movement with the inside surface of the foot is considered one of the movements of open motor

sequences that belong to the circular movement aiming at producing high angular velocity for the kicking leg 
through the kinematic form of different body links taking part in the performance. Passing curved balls in effective places in front of the opponent goal on purpose to give chances for direct shooting at the goal is one of the strategies used during carrying out the corner kick. (10)

Direction of the rotation of the ball is outlined according to the place of its pass as it is preferable to play it in a curved line to the contrary of the goal when passing it for a long distance to the far area of the executive angle to enable forwards to head the ball easily thus increasing the shot strength at the goal, whereas, it is preferable to play the ball in a curved form in the direction of the goal when passing it for a short distance in front of the close post to help forwards who don't face the goal to direct the ball with the back of the head towards the goal directly or to make a cross in front of the goal. (8)

Research problem:

Through his follow-up of different local and international Soccer matches the researcher found that the rate of corner kicks towards the close post of the goal and passing away from the executive angle were increased as these places were more serious at the goal of the opponent team. For the importance of passing corner kicks precisely to such effective areas, it was difficult for the performing player to pass the ball to the assigned place with the required specifications in respect of its strength, velocity, precision of motor pathway to be received by a forward as required to shoot it at the goal directly or to pass it to another forward in a better position to complete the attack. Since kinematic properties of different body parts were different during carrying out corner kicks because of directing corner kicks to different places according to forwards' planning movements previously agreed, it was difficult for the player executing the performance to evaluate requirements of the best performance correctly to each place where he wanted to play the ball through it precisely. The simplest change especially in the correct evaluation of any kinematic 
property of any body part during the performance would result in not achieving the aim of the corner kick and the ball would go away from the intended place that the ball should reach in and consequently, the corner kick would lose its effect due to the increase of the defensive concentration at the goal.

Aim of the research:

In this context the research objective was outlined to compare differences among kinematic properties such as displacement and linear velocities and angles and angular velocities during performing corner kicks for Junior Soccer players with techniques of ball rotation towards the goal in the close post area and to the contrary of the goal in the rear area at the moment of launching the ball.

Research Methodology:

The researcher applied the descriptive method with the comparative analytical technique and using video depending on the Simi Motion program for 3D motor analysis.

The research Sample:

It was selected intentionally from Al-Sharkia Sporting Clubs including (3) players who were the best in playing corner kicks according to the test of corner kicks performance precision to specific areas in front of the goal with the right foot. (10) Trials were shot with video for each technique. The research sample was characterized as the skewness coefficients of averages of growth and training age ranged from (0.50 to 1.629$)$ indicating that data were normal when performing corner kicks according to ball rotation technique towards the goal.

\section{Tools of collecting data:}

- Legal Soccer playground of $100 \mathrm{~m}$ length of the goal line.

- Legal foot balls.

- Calibration box of $1 \mathrm{~m}$ of its different dimensions.

- A number of (2) 3D camera tripod.

- A number of (2) video camera of brand Fastec Imaging of 250 frames/sec with operating cables.

- 3D Simi Motion Analysis System with its operating requirements.

Test of precision of performance of corner kicks:

To measure the precision of corner kicks according to 
ball rotation towards the goal technique the researcher reviewed several references and previous studies introducing measuring the precision of performance of corner kicks and he depended on the test of the precision of passing the corner kick in front of the goal, which has been proved that it had higher reliability and stability coefficients via the study of Louay Ghanem and Nasha'at Bashir (2012)(8) as the test gained stability of 0.869 whereas its self-reliability was (0.932). The researcher used this test on purpose to outline the best correct trials with the right foot for each player in the research sample and in each type of corner kicks as per the ball rotation technique. The researcher excluded trials giving inaccurate data during motor analysis due to faulty imaging or performance. The result of this process was outlining (10) correct trials for each type of corners as per the ball rotation.

\section{Motor analysis program:}

The researcher carried out imaging and motor analysis in coordination with Sports Consultancies and Researches
Center, Faculty of Physical Education, Zagazig University using Simi Motion Program for motor analysis designed to track and to analyze the motion. The researcher used this program for several reasons: It is characterized by multiple mechanical indices extracting their results so precisely, instantly recording the movement without stop during the performance, possibly analyzing with one or more cameras for the movement of the player's body as a whole or an outlined part of it, no need of complex calibration system and imaging could be done inside halls or open places.

\section{Pilot study:}

The researcher carried out the pilot study on Sunday, 19/2/2017 at Zagazig University Stadium and in coordination with

Consultancies and Researches Center, Faculty of Physical Education, Zagazig University on a sample of (3) Junior players from Al-Sharkia Sporting Club under (18) years from the research community but outside the main research sample and characterized by performing corner kicks 
correctly. The results concluded as follows:

- Assuring of the legality of the playground particularly the goal and corner areas.

- Outlining the position of the calibration box used in the analysis precisely for each type of corners to make sure that the ball was existed during the performance in the same place approximately during each of the two types.

- Outlining the place of putting cameras during performing the corner kick of the close post with the ball rotation technique towards the goal provided that an angle of 900should be made between them. Camera (1) should be away from the post of corner flag with $8 \mathrm{~m}$ distance on the touch line. Camera (2) should be the same distance on the goal line.

- Putting cameras during performing the corner of the rear area with the ball rotation technique in contrary of the goal direction with also (900) angle between them, as camera (1) is put on the extension of the touch line outside the playground behind the goal line with (8) $\mathrm{m}$. distance of the post of the corner flag. Camera (2) should be put on the goal line provided that it should be away from the post of the corner flag with also (8) $\mathrm{m}$.

- Cameras should be mounted on tripods at $(125 \mathrm{~cm})$ height from the ground surface.

- Making sure that cameras were synchronized at 250 frame/sec.

- Imaging was outlined to start at 3:00 pm.

- Practicing assistants on procedures of imaging and making sure that they understood the research nature.

- Making suitable warmup before performing the test of precision of passing the corner kick to the head with the solenoid technique.

- The player should perform (4) correct trials in each type of corners with enough rest interval between them.

- $\quad$ Imaging one trial in each type of corners and identifying the method of analyzing its data in coordination with the Sports Consultancies Center at the college by using Simi Motion Program. 


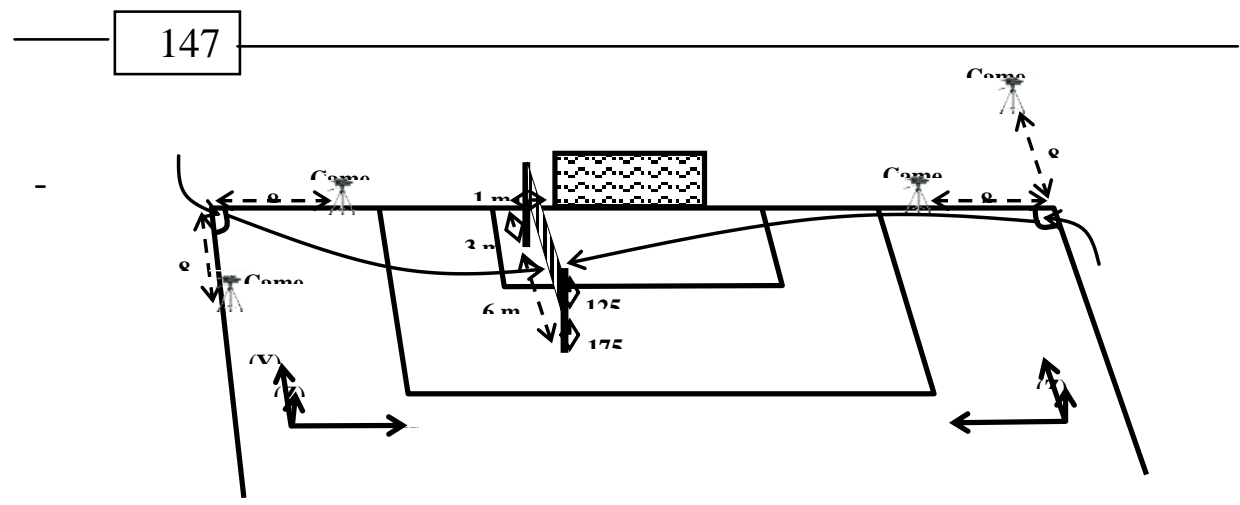

Fig. (1): Coordinates and positions of placing cameras during performing corner kicks with their two types according to the ball rotation technique towards the goal Main study:

After the pilot study had achieved its objectives, the researcher knew problems that could face and challenge him, he overcame them in limits of possibilities available, he concluded the final steps of imaging and he conducted the main study on $2 / 3 / 2017$ at Zagazig University Stadium on the main research sample as (10) trials were imaged for each corner kick and he used the Simi Motion Program to analyze their results according to the following steps:

- Reviewing video shots with cameras used for each trail and outlining numbers of each of them during analysis provided that each type of the corner kicks should be stored in a special folder.

The calibration system should be stored in the memory of the computer for each type of the research corner kicks.

- $\quad$ Selecting points of body parts that their kinematic properties would be analyzed by motor analysis unit as the required file would be recalled to carry out linear and angular accounts for points to be computed in moments assigned during performing the skill as the researcher defined the moments of maximum suppressing and launching the ball to express the skill performance.

- The program would compute values of such properties after giving the order to do so as linear velocity and displacement and angles and angular velocities were computed for different body parts. 


\section{8}

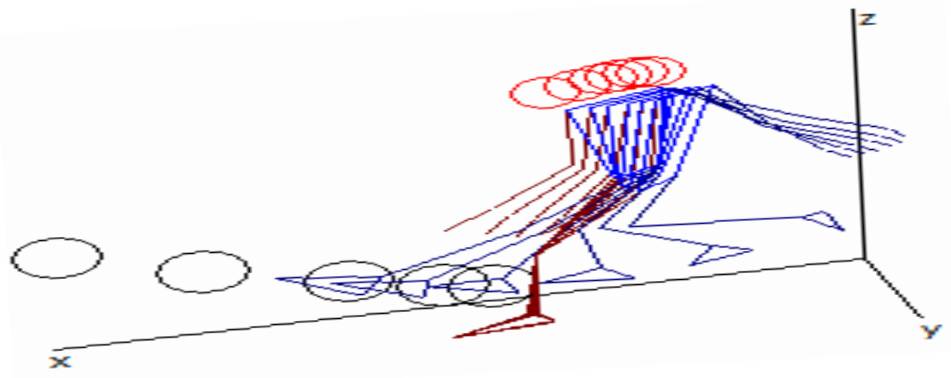

Fig. (2): Stick like forms of corner kicks.

Results and discussion:

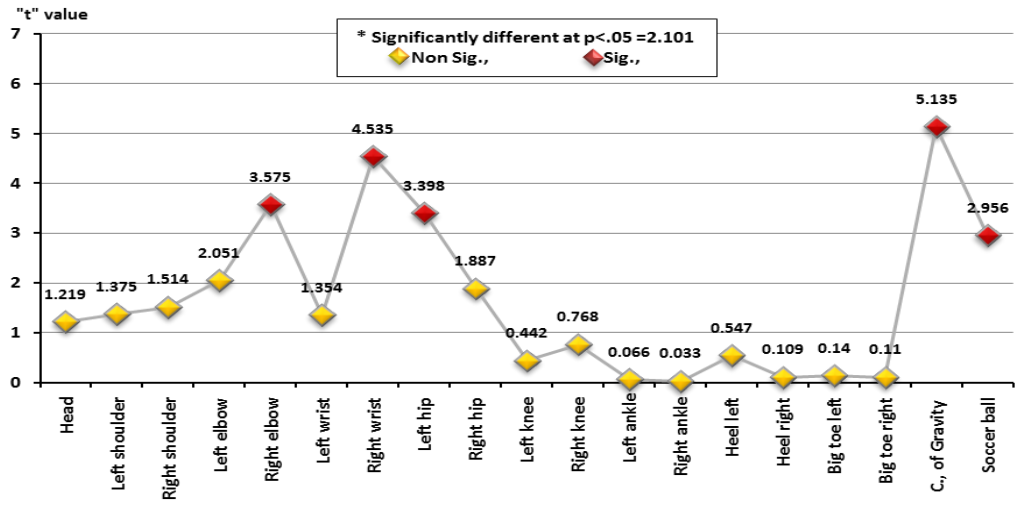

Fig., (3): Significance of differences between horizontal Displacements of body parts during performing corner kicks for the close post and beyond area at launching the ball.

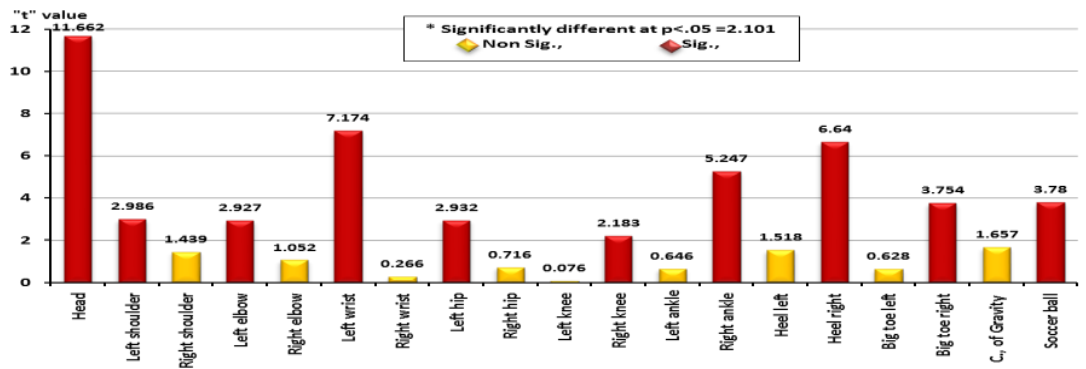

Fig., (4): Significance of differences between horizontal velocities of body parts during performing corner kicks for the close post and beyond area at launching the ball.

Data in Figures (3) (4) horizontal velocities of points indicate that there are of the kicking leg represented significant differences in by knee, ankle and the right 
heel at the moment of launching the ball in favor of the corner kick of the beyond area and the right instep in favor of the corner kick of the close post. The researcher attributed the superiority of the corner kick of the beyond area in horizontal velocities of knee, ankle, and the right heel to increase the rush and velocity of swinging the right leg kicking forward at the moment of kicking and launching the ball as it is considered as one of the final links that all moments of forces move to it to produce more strength when kicking the ball, the far distance of the beyond area in comparison with the close post area to the ball and thus increasing the velocity of such points leading to increase the amount of strength added by these parts to the ball at the moment of its launching. It is also noticeable that the horizontal velocity of such parts is ascending meaning that the horizontal velocity of the right knee is less than that of the right ankle which in its turn is less than the velocity of the right heel as a reasonable result of its point preceding the other from the top to the bottom as an axe of rotation for the following point and hence, its velocity becomes lower than it because of gaining more motion and so the right heel point has the highest horizontal velocity in the types of two corner kicks and having the biggest difference in favor of the corner kick of the beyond area. The same data also illustrate that there are significant differences in the horizontal displacement at the moment of launching it in favor of the corner kick of the beyond area and the researcher attributed that to the range of strength gained from the velocity of the right foot while swinging forward as the results also show that the velocity of the kicking foot (instep, heel and ankle) is increased in all different horizontal, cross or vertical directions of the motion in favor of the corner kick of the behind area helping such strengths move farther horizontal distance than that of the corner kick of the close post at the moment of freeing the ball from the kicking foot as that agreed with the study of Alen Kapidzic et al., (2014) (1) that the most important reason for increasing ball velocity and its moving forward during the performance in Soccer was the high velocity achieved by the kicking foot at the moment of collision with the ball. 
While the researcher thought that the superiority of the corner kick of the close post in horizontal velocity of the right instep at the moment of launching the ball could be related to the increase in moving the right instep upward at the moment of its launching in corner kick of the behind corner and thus increasing vertical velocity in a greater form and in contrast the horizontal velocity of the right instep is increased and its vertical velocity is decreased through the corner kick of the close post for two reasonsfirst, the right instep moves forward and outside at the moment of contacting the ball to make it gain the necessary rotation towards the goal and hence, its horizontal velocity is increased while the far distance of the close post area does not allow more vertical velocity for the instep upward and hence, we find that the angle of the right ankle is more during the corner kick of the close post.

On the basis of the clear differences shown from the results in total of horizontal velocity of the right leg points at the moment of launching the ball in favor of the beyond area, this helps increase the horizontal velocity the ball gained as a final output for performance when kicking with the foot inside surface for farther distance. Hesham Hendawy et al., (2010)(2) indicated that the velocity and angle of launching the ball and the angular velocity of the kicking foot affected directly outlining the position of dropping the ball and the success of performing the corner kick.

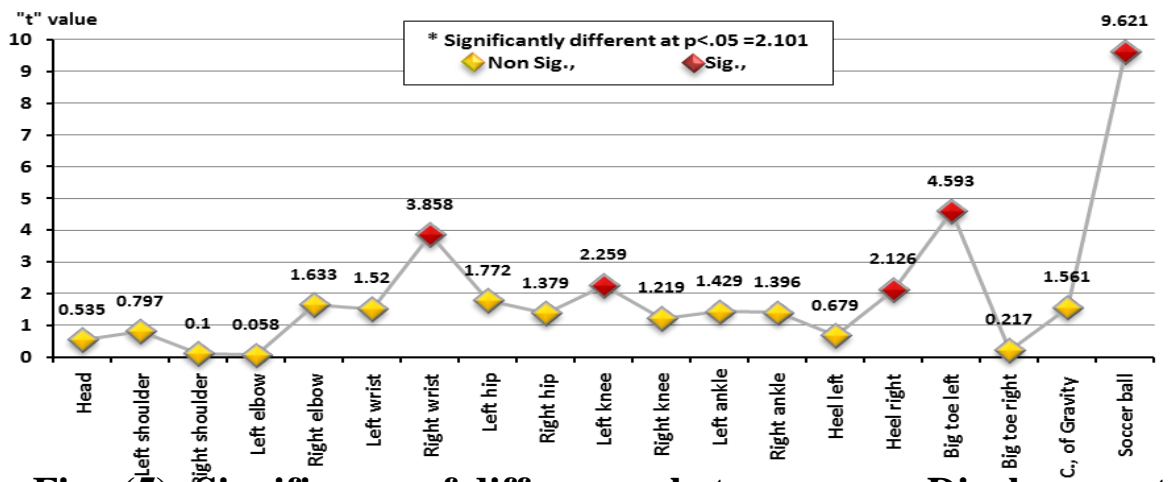

Fig., (5): Significance of differences between cross Displacements of body parts during performing corner kicks for the close post and beyond area at launching the ball. 


\section{1}

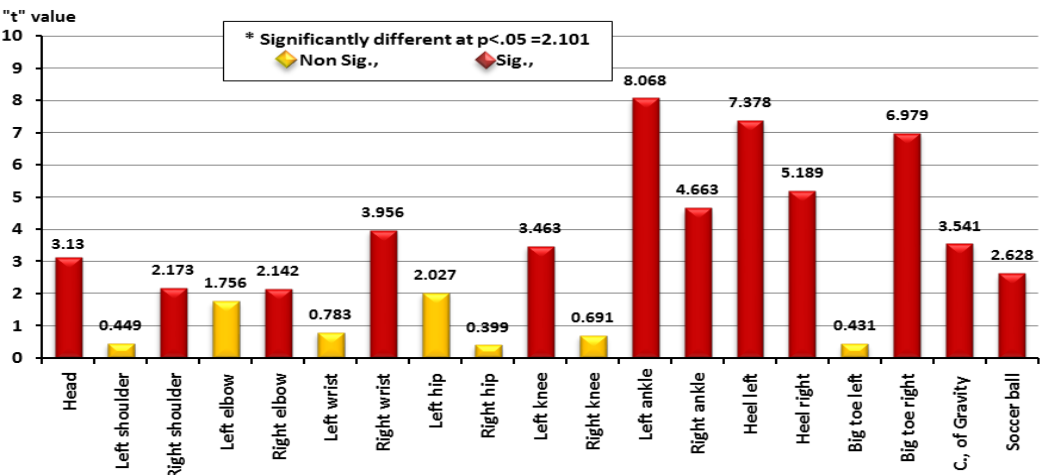

Fig., (6): Significance of differences between cross velocities of body parts during performing corner kicks for the close post and beyond area at launching the ball.

Data in Figures (5) (6)

illustrate that there are significant differences in the cross displacement of knee and instep of the left foot at the moment of launching the ball in favor of the corner kick of the beyond area and the researcher attributed this result to the knee moved more outward during corner kick of the beyond area to have the kicking foot to move freely at the cross section and to face the ball correctly. While the researcher attributed the significant differences of the cross displacement of the left instep in favor of the beyond area to the kicking technique during this type of corner kick that considerably depended on the ball rotation in contrary to the goal direction and to direct the ball to the beyond area by this technique the left instep should be directed during the phase of maximum damping outward the body allowing to kick the ball at the moment of its launching toward the outlined position. In the meantime, the cross displacement of the left instep is lower during the corner kick of the close post due to the ball rotation technique toward the goal that is in need of directing the left instep in the phase of maximum damping inward the body slightly allowing to launch the ball after swinging the kicking leg in its direction and directing it to the outlined position. The results also indicate that there are significant differences in the cross displacement of the right heel at the moment of launching the ball in favor of 
the corner kick of the close post and the researcher related that to the ball rotation technique toward the goal in types of corner kicks as the ball rotation technique to the contrary of the goal direction in the corner kick of the beyond area needed the heel of the right foot to move outward more than that of the corner kick of the close post decreasing its cross displacement at the moment of kicking and launching the ball. Lees et al., (2010)(7) illustrated that cross displacements occurred in the right foot because of twisting the foot outward to have the ball to go out in an arc form and hence, increasing the cross displacement of the right foot the produced strength would be more.

It is clear that there are significant differences in cross velocities of knee, ankle and heel of the left foot at the moment of launching the ball in favor of the corner kick of the beyond area and the researcher related that to the ball kicking technique to the contrary of the goal direction needing to twist the instep of the left foot outward the body at the moment of maximum damping making the cross displacement of knee, ankle and heel of the left foot more in the corner kick of the beyond area making the side slope of the kicking leg in general more in that corner kick. Jin Wang and Mike Griffin (1997)(4) indicated that swinging the right leg aimed at increasing the amount of angular movement as the pelvic contracted and turned left slightly then the right joint of the pelvic started contracting followed by extending the right knee resulting in outcome of strengths and maximum angular velocity for the foot at the moment of collision with the ball.

Whereas the researcher observes that there are no significant differences in cross velocity for the left instep at the moment of launching the ball due to relative stability of the instep as it is considered an essential foundation in the process of braking the body and preventing it from rushing forward or aside at the moment of kicking the ball in corner kicks of the research particularly the left instep is directed to the position of 
passing the ball as it is directed inward the body during the corner kick of the close post and outward during the corner kick of the beyond area with the result that the heel and ankle of the left foot moves in contrary to the direction of moving the instep and hence a strong base is occurred from the left foot that helps balance the player's body at the moment of launching the ball and preventing him from dropping aside and directing the ball precisely to the intended position while the cross velocity of ankle, heel and the right instep at the moment of launching the ball in favor of the corner kick of the close post. The researcher attributed that to the increase in the cross displacement of such points considerably in the corner kick of the close post at the moment of maximum damping because it was difficult for the body to move forward in the cross direction as compared to the corner kick of the beyond area, consequently, the motor range was increased of such points at the cross level (side moving) at the moment of the maximum damping till the moment of launching the ball and hence, the cross velocity of the ball was increased in the corner kick of the close post. The researcher attributed the presence of significant differences in the cross velocity of the ball at the moment of its launching in favor of the corner kick of the close post to the ball rotation technique towards the goal where the ball was moving at the moment of its launching in a curved path making a larger angle with the goal line than that of the corner kick of the beyond area where the ball would move in a rotational form in the cross direction to the contrary of the goal direction and hence, the amount of cross movement and then the cross velocity would be larger in the corner kick of the close post, whereas the ball rotation technique to the contrary of the goal direction was characterized by increasing horizontal and vertical velocities at the moment of its launching as a result of the far distance of the beyond area from the ball position that required increasing the horizontal velocity which in turn 
increasing the height of the ball within its path to allow the ball to drop in the outlined area. Pekka Iuhtanen (2002) (10) indicated that variance of direction and velocity of the ball depending on the position of collision of the kicking leg with the ball as the external rotation of the ball created different air pressure on both sides of the ball and the ball is moved from the high pressure at the external side to the lower pressure at the other side to follow the curved path to reach its outlined position in front of the goal.

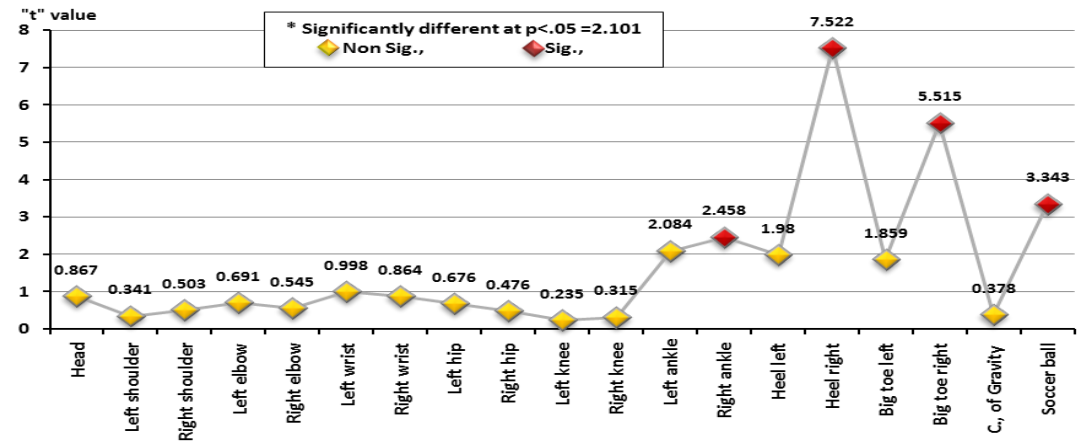

Fig., (7): Significance of differences between vertical Displacements of body parts during performing corner kicks for the close post and beyond area at launching the ball.

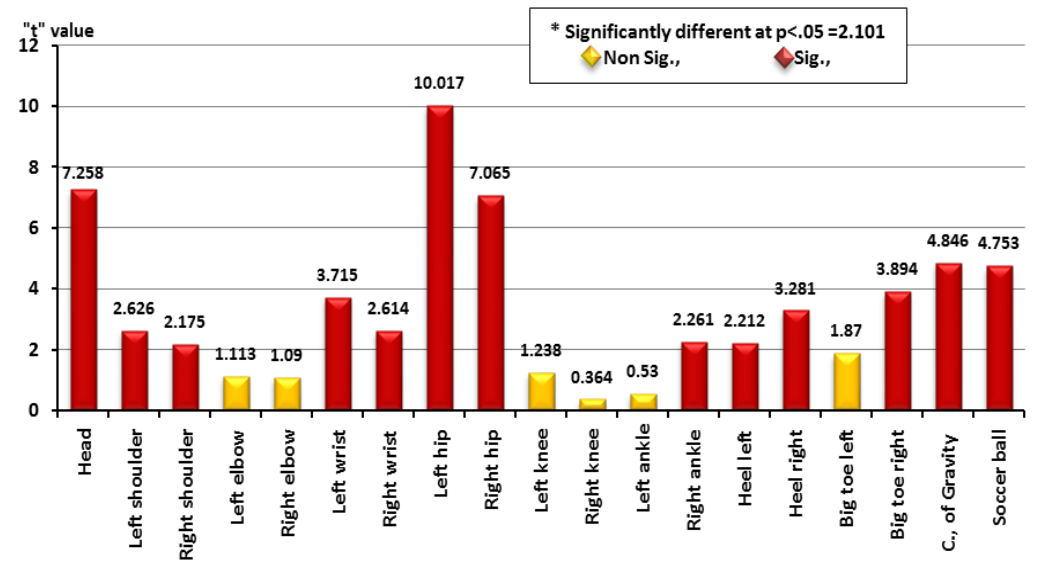

Fig., (8): Significance of differences between vertical velocities of body parts during performing corner kicks for the close post and beyond area at launching the ball. 


\section{5}

Data in Figures (7) (8) demonstrate that there are significant differences in vertical displacements of ankle, heel and instep of the right foot at the moment of launching the ball in favor of the corner kick of the beyond area and the researcher attributed these results to direct the ball precisely that depended on directing ankle and heel of the right foot to a point below the ball center and outward from the right side of the ball and hence, the ankle and heel of the right foot would be responsible for the ball rotation when touched from the outside and also responsible for the ball height when touched below its center by the assistance of moving the instep of the right foot upward during kicking the ball and consequently, raising the ankle and heel of the right foot in the corner kick of the beyond area would give a greater chance to move the instep of the right foot upward during kicking the ball increasing the ball launching angle. Also, the significant differences in vertical displacement of the ball at the moment of its launching in favor of the corner kick of the beyond area indicate that the ball is higher from the ground in comparison with the corner kick of the close post due to the velocity of swinging the right foot kicking from back to front in each of the right ankle and heel and transferring this horizontal velocity to an increase in the vertical velocity of the instep of the right foot at the moment of its moving upward during kicking the ball making the joint of the right ankle be in a less angle than the corner kick of the close post and hence, the ball is raised more from the ground. Lees, A. T. et al., (2010) (7) indicated that the player while kicking with the inside foot surface tightened the joint of the right ankle upward at the moment of collision with the ball and hence increasing the strength of the ball exited from the player.

Although there are no significant differences between the research corner kicks in vertical displacement of the heel of the left foot at the moment of launching the ball, there are significant differences in vertical velocities in favor of the corner kick of the beyond area. The researcher attributed 
that to increase the horizontal velocity of the right foot while swinging it forward when kicking the ball and hence making the heel of the left foot move upward slightly with a vertical velocity to brake the movement of the player's body and this is shown clearly in the corner kick of the beyond area as a result of movement of the instep of the right foot kicking upward more than that in the corner kick of the close post while touching the ball making the heel of the left foot raise from the ground to transfer moments to great links like pelvis and in turn to the right leg kicking and hence, significant differences appear in vertical velocities of right and left pelvis in favor of the corner kick of the beyond area. Also the researcher attributed the presence of significant differences in vertical velocities of the instep kicking at the moment of launching the ball in favor of the corner kick of the beyond area to the right foot instep that continued to move vertically high at the moment of touching the ball in the corner kick of the beyond area whereas it moved forward in the corner kick of the close post as a result of the difference in the performance technique of each type of corner kicks and that helped the corner kick of the beyond area be superior in the vertical velocity of the instep of the right foot while the corner kick of the close post was superior in horizontal velocity and consequently, vertical velocities of the right ankle and heel were decreased as points or axes of turning the instep of the right foot upward in the corner kick of the beyond area and hence, the corner kick of the close post was superior in vertical velocities of such points and considering the amount of ball velocity was the final target of performing corner kicks, the horizontal and vertical velocities should be considered as an important indicator of the strength and precision of corner kicks toward outlined positions in front of the goal. Therefore, the results show clear superiority in the horizontal and vertical velocities in favor of the corner of the beyond area, in the meantime, the corner of the close post is superior in cross velocities as a result of the ball rotation technique toward the 
goal which needs a cross launching angle located between the side path of ball displacement and goal line from the position of the ball as this angle is increased more when compared to the ball rotation technique in contrary to the goal direction. Louay
Ghanem and Nasha'at Bashir (2012) (8) indicated that there were significant differences in maximum deviation of the ball to the goal line at the moment of kicking the ball in favor of corner kick toward areas of less distance.

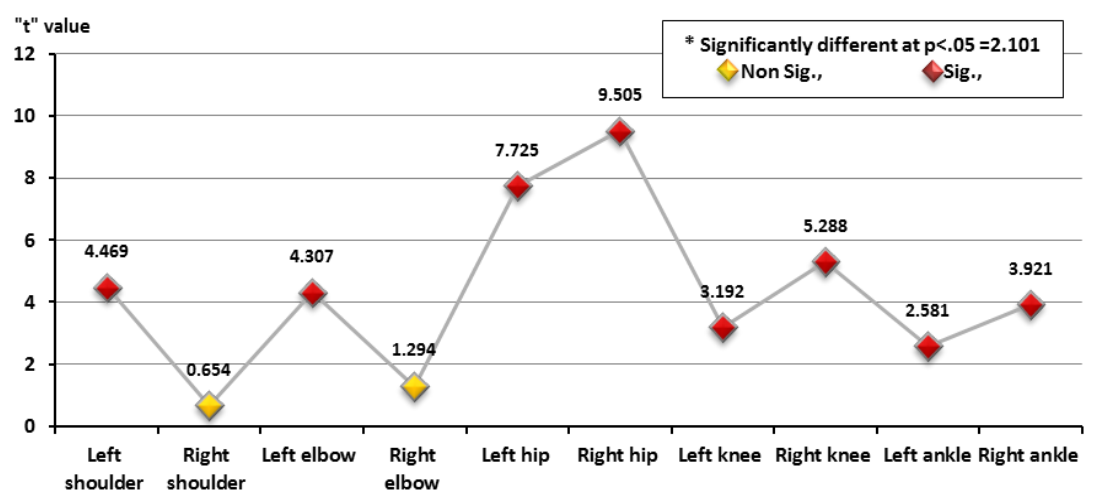

Fig., (9): Significance of differences between Angles of body parts during performing corner kicks for the close post and beyond area at launching the ball.

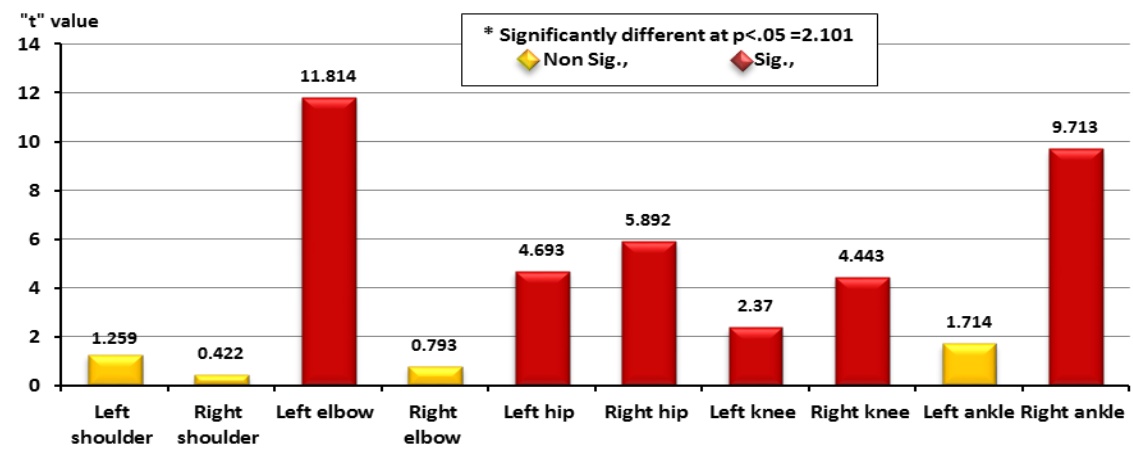

Fig., (10): Significance of differences between angular velocities of body parts during performing corner kicks for the close post and beyond area at launching the ball. 
Data in Figures (9) (10) clarify that there are significant differences in angles and angular velocities of knee and the left pelvis in favor of the corner kick of the beyond area as the extension of the left knee via the movement of the left hip upward in the research corner kicks helped increase the angle of the left knee in each type of corner kicks and it was more in the corner kick of the beyond area leading to increase its angular velocity greatly affecting the decrease in the angle of the left pelvis as a result of moving the left torso forward in corner kick of the beyond area and the increase of the same angle as a result of moving the left torso in the corner kick of the close post at the moment of launching the ball. Koichiro Inoue (2014)(5) indicated that the left knee was near to the full extension at the moment of launching the ball as a result of the process of motor transfer of strength from the positioning foot to the foot kicking which in its turn moved forward to transfer the body center of gravity from the fixed foot to the moving foot which in its turn contributed to increase gravity of the swinging foot during the performance.

Despite the presence of significant differences in the vertical velocity of the left heel and the angle of the left ankle in favor of the corner kick of the beyond area, but such differences do not associate with the significant differences in the angular velocity of the left ankle for the movement of the left leg forward and upward when the left heel leave the ground toward upward while the left instep is still in touch with the ground in a state of relative stability as the body does not rush forward or upward at the moment of launching the ball and consequently, the angular velocity is decreased in the research corner kicks to control different vertical velocities and not to imbalance of the body during the performance. Lees, A. and Nolan, L. (1998) (6) stated that the positioning foot had an important role in braking the movement of the 
body particularly the instep of left foot that helped have the left knee to be on the same line with the hip and to move upward in the vertical direction by raising the heel of the left foot from the ground gaining the body the necessary brake to prevent the body imbalance.

The results show that there are significant differences in angle and angular velocity of the right knee in favor of the corner kick of the beyond area at the moment of launching the ball due to the movement of the right leg and foot forward and downward more quickly with the result that the angle of the right knee is increased considerably more than the corner kick of the close post as the increase in such velocity during swinging the right leg forward makes the right foot more strength during the performance and hence achieving the purpose of kicking with the inside foot surface for further distance whereas the significant differences are in favor of the corner kick of the close post by increasing the angle of the right ankle due to the movement of the instep of the right foot at the moment of kicking the ball forward and downward while moving forward and upward in the corner kick of the beyond area to decrease the angle of the right ankle with the result that the vertical velocity of launching the ball is increased making the ball raise vertically and considerably as Mohamed Abdel Hamid (2002) (9) stated that the ankle and heel of the right foot are off the center of the ball downward slightly to give a chance to the instep of the kicking foot to twist around the ball to save the strength and precision.

\section{Conclusions:}

- Movement of the instep of the right foot upward in the corner kick of the beyond area contributed to increase the vertical velocity with lessening the angle of the right ankle considerably whereas the movement of the instep of the right foot forward and outward in the corner kick of the close post increase its horizontal and cross velocities considerably. 
- Directing the ball to the beyond area with the ball rotation technique in the contrary to the goal direction needs the knee and instep of the left foot to move outward whereas such points should move inward slightly in the corner kick of the close post with the result that significant differences between them are existed in the cross displacements of such points.

The ball rotation technique in contrary to the goal direction needs the heel of the right foot to move outward considerably with the result that the cross displacement is decreased in comparison with the corner kick of the close post where such displacement is increased causing its cross velocity to increase for points of the right foot more than that of the corner kick of the beyond area.

Comparing differences among cross displacements between the heel and the instep of the right foot, the right foot is twisted inward considerably in the corner kick of the beyond area because of the ball rotation technique.

- The corner kick of the close post is superior in the cross velocity of the ball as the curved path of the ball is making a greater side angle with the goal line whereas the corner kick of the beyond area is superior in horizontal and vertical velocities of the ball due to the distance of the place of the beyond area from the position of the ball.

- The increase in vertical displacements of the ankle, heel and instep of the right foot in the corner kick of the beyond area contributes to move the instep of the right foot upward while kicking the ball causing its vertical velocity to increase and hence, the angle of launching the ball is increased and the path of the ball gets higher than that in the corner kick of the close post.

- The left knee extension via moving the left hip upward helped increase the angle of the left knee considerably in the corner kick of the beyond area with the result that its angular velocity is increased. 
- $\quad$ The velocity of swinging the right leg forward caused the angle of the right knee to increase considerably in the corner kick of the beyond area, whereas the velocity of moving the instep of the right foot forward and downward helped the angle of the right ankle increase considerably in the corner kick of the close post.

\section{Recommendations:}

- Building educational and training programs depending on the optimum rate of kinematic characteristics when performing corner kicks with its different type.

- Establishing quality exercises that help increase the velocity of the kicking leg as required when performing corner kicks according to the ball rotation technique toward the goal in each type.

- Considering the process of positioning the foot supporting as it is important in damping the velocity of rushing the body forward and transferring moments of the kicking leg and keeping the body balance.
- Working on extracting predictive equations to expect the precision of passing corner kicks with its different type according to important kinematic contributing characteristics performance.

\section{References:}

1- Alen Kapidžić, Tarik Huremović, Alija Biberovic (2014) : Kinematic analysis of the instep kick in youth Soccer players , J., Human Kinetics volume 42.

2- Hesham Hendawy, Rafat abd al-Hady and Mohaned yaser (2010): Predicting of dropping balls of corner kicks in Soccer in the light of biomechanical variables. A research published in Journal of Al-Qadisiyah of Sciences of Physical Education. Vol. 11, Special Issue for the 2nd Scientific Conference on Biomechanics Dec. 25 - 26.

3- Hanafy Moktar (1994) : Scientific Fundamentals in Practicing Soccer . Dar Al-Fekr Al-Arabi., Cairo.

4- Jin Wang and Mike Griffin (1997): Kinematic analysis of the soccer curve 


\section{2}

Ball Kick , J., national strength and conditioning Association, Vol., 19, No.,1.

5- Koichiro Inoue (2014):

Dynamics of the support leg in soccer instep kicking, j., of sports sciences, Routledge, England.

6- Lees A., and Nolan L., (1998): The biomechanics of soccer A review, j., of sports sciences, Routledge, England.

7- Lees A., Asai B.. Andersen H., Nunome T., Sterzing (2010): The biomechanics of kicking in soccer : review, J., of Sports Sciences, Routledge , England.

8- Louay Ghanem and Nasha'at Bashir (2012): L Analyzing some Mechanical Variables of Corner Kick with
Spiral Technique in Contrary to the Goal in Soccer . Journal of Al Qadisiyah of Sciences of Physical Education, Vol. 13. Special Issue for Proceedings of the 3rd Scientific Conference on Biomechanics, Feb. 5

\section{9- Mohamed Abdel Hamid} (2002): A biomechanical comparison between kicking with the inside and front foot surfaces in Soccer, Journal of Theories and Applications of Faculty of Physical Education for Boys, Alex. University. 10- Pekka luhtanen (2002): Biomechanical Aspects of soccer performance, The Faculty of education, Edinburgh university. 